\title{
A Convex Parameterization of All Stabilizing Controllers for Non-Strongly Stabilizable Plants under Quadratically Invariant Sparsity Constraints
}

\author{
Şerban Sabău and Nuno C. Martins
}

\begin{abstract}
This paper addresses the design of controllers, subject to sparsity constraints, for linear and time-invariant plants. Prior results have shown that a class of stabilizing controllers, satisfying a given sparsity constraint, admits a convex representation of the Youla-type, provided that the sparsity constraints imposed on the controller satisfy a certain condition (named quadratic invariance) with respect to the plant and that the plant is strongly stabilizable. Another important aspect of the aforementioned results is that the sparsity constraints on the controller can be recast as convex constraints on the Youla parameter, which makes this approach suitable for optimization using norm-based costs. In this paper, we extend these previous results to the general case of possibly non-strongly stabilizable plants. Our extension is conveyed in terms of a parametrization for the class of controllers that is very similar to the Youla parametrization. In our extension, under quadratically invariant constraints, the controller class also admits a representation where the free parameter is subject to only convex constraints. While the strong stabilizability assumption has been removed our result yields the same elegant simplicity from the strongly stabilizable case.
\end{abstract}

\section{INTRODUCTION}

The design of optimal, decentralized control systems is in general a hard problem, partly due to lack of convexity induced by restrictions on the structure of the controller. Typically, these constraints arise from pre-specified information patterns, such as when the controller consists of interconnected blocks that have access only to certain measurements.

The theoretical machinery developed in [6] unifies and consolidates many previous results, pinpoints certain tractable decentralized control structures, and outlines the most general known class of convex problems in decentralized control. The authors of [6], propose a computational procedure for the design of $\mathcal{H}^{2}$ optimal, decentralized controllers of strongly stabilizable plants under quadratically invariant structured constraints. This paper is an extension of the method from [6] to the general case of possibly nonstrongly stabilizable plants.

Notice that necessary and sufficient conditions for strong stabilizability, of general, multi-input multi-output, linear, time-invariant plants, are not yet known in the literature. Neither are general methods for designing stabilizing controllers that are stable, for the cases in which they do

Ş. Sabău is with the Electrical and Computer Engineering Dept. Univ. of Maryland at College Park, A.V. Williams Bldg., Rm. 2248, College Park, MD 20742-3285, USA email: sserban@isr.umd.edu. Şerban Sabău was supported by NSF CMMI award number 0901210

N. C. Martins is with the Faculty of the Electrical and Computer Engineering Dept. and the Institute for Systems Research, University of Maryland, College Park, MD 20742-3285, USA. email: nmartins@isr.umd.edu. Nuno C Martins was supported by AFOSR award number FA95500810120 and NSF ECCS award number 0644764. exist. This makes designing stable controllers for strongly stabilizable plants a difficult task even in the centralized setting. More importantly, for most practical situations in control engineering, the working hypothesis is stabilizability only, rather than strong-stabilizability.

For the design method in [6], the optimal controller (in the $\mathcal{H}^{2}$ sense, for instance) can be synthesized via convex programming, starting from a stable, stabilizing controller. While inheriting this feature, our approach has the increased handiness of relying on just any stabilizable controller, not necessarily stable, which in general is far easier to find. This bridges the gap between stability constraints and the main optimization paradigm, hence it has the merit of not overcomplicating the final convex program with additional tough constraints related to stabilization.

The core of our approach resides in the so called coordinate-free method proposed in [4], where coprime factorizability of the plant is not needed, to provide a parametrization of all stabilizing controllers. Using this parametrization and much in the spirit of [6], our main result shows how to deal with the decentralized problem for quadratic invariant structures, provided the availability of tools to solve the centralized problem.

For a more detailed treatment of the results presented in this paper, we refer to [10].

\section{Preliminaries}

It followed quite naturally to develop our results over any ring of stable, linear systems, within the general framework established in the seminal paper [8] of Vidyasagar et al. Complying with [8, Definition 3.1], the notions of causal and strictly causal are introduced in an abstract setting, and any transfer function is viewed as the ratio of two stable, causal transfer functions. The advantage of using this setup is that it encompasses within a single framework, continuous or discrete-time systems, lumped as well as distributed systems, $n-D$ systems, etc. The important special case of linear, time-invariant, $1-D$ systems, is immediately retrieved by considering the instance of the ring of proper, stable, rational functions.

With all the notation borrowed from [4], $\mathcal{A}$ is the set of stable, causal transfer functions and is assumed to have a commutative ring structure.

The set of all transfer functions, which we denote as $\mathcal{F}$, is therefore the field of fractions of $\mathcal{A}$, defined as follows:

$$
\mathcal{F} \stackrel{\text { def }}{=}\{n / d \mid n, d \in \mathcal{A} ; d \text { not a divisor of zero }\}
$$


Accordingly, $\mathcal{F}^{p \times m}$ stands for the set of transfer function matrices (matrices with all entries in $\mathcal{F}$ ) with $p$ rows and $m$ columns. Let $\mathcal{Z}$ be any be any prime ideal of $\mathcal{A}$ with $\mathcal{A} \neq \mathcal{Z}$ and such that $\mathcal{Z}$ includes all the divisors of zero of $\mathcal{A}$. Define the subsets $\mathcal{P}$ and $\mathcal{P}_{s}$ of $\mathcal{F}$ as

$$
\begin{gathered}
\mathcal{P} \stackrel{\text { def }}{=}\left\{\frac{a}{b} \in \mathcal{F} \mid a \in \mathcal{A}, b \in \mathcal{A}-\mathcal{Z}\right\}, \\
\mathcal{P}_{s} \stackrel{\text { def }}{=}\left\{\frac{a}{b} \in \mathcal{F} \mid a \in \mathcal{Z}, b \in \mathcal{A}-\mathcal{Z}\right\} .
\end{gathered}
$$

We shall call every transfer function in $\mathcal{P}\left(\mathcal{P}_{s}\right)$ causal (strictly causal). Similarly, if every entry of some transfer function matrix is in $\mathcal{P}\left(\mathcal{P}_{s}\right)$ then the transfer matrix will be called causal (strictly causal).

\section{Parametrization of Stabilizing Controllers VIA THE COORDINATE-FreE APPROACH}

In [1, Figure 3.2, pp.15] the standard feedback interconnection between a generalized plant and a controller is depicted. There, $w$ is the vector of reference signals, while $\nu_{1}$ and $\nu_{2}$ are the disturbance signals and sensor noise respectively. In addition, $u$ are the controls, $y$ are the measurements and $z$ the regulated outputs (in general some error signals). For convenience of notation, $G$ is partitioned accordingly with $G_{z w} \in \mathcal{F}^{n_{z} \times n_{w}}, G_{z u} \in \mathcal{F}^{n_{z} \times n_{u}}, G_{y w} \in \mathcal{F}^{n_{y} \times n_{w}}$ and $G_{y u} \in \mathcal{F}^{n_{y} \times n_{u}}$. Here, the integers $n_{w}, n_{u}, n_{y}$ and $n_{z}$ denote the dimensions of $w, u, y$ and $z$ respectively. The generalized plant $G$ lies in $\mathcal{F}^{\left(n_{y}+n_{z}\right) \times\left(n_{u}+n_{w}\right)}$ and the controller in $\mathcal{F}^{n_{u} \times n_{y}}$. We adopt the superscript $T$ as the notation for matrix transposition. Assuming that the loop is well posed - that is $\left(I-K G_{y u}\right)$ is invertible over $\mathcal{F}^{n_{u} \times n_{u}}$ - then the transfer matrix from $\left[\begin{array}{lll}w^{T} & \nu_{1}^{T} & \nu_{2}^{T}\end{array}\right]^{T}$ to $\left[\begin{array}{lll}z^{T} & u^{T} & y^{T}\end{array}\right]^{T}$ is denoted by $\Theta(G, K)$.

If the transfer matrix $\Theta(G, K)$ is over $\mathcal{A}$ then we call it stable, or we say that $K$ is a a stabilizing controller of $G$, or equivalently that $K$ stabilizes $G$. If a stabilizing controller of $G$ exists, we say that $G$ is stabilizable.

Of particular interest is the feedback system displayed in [1, Figure 1.1, pp.2], where the transfer function matrices $K \in \mathcal{F}^{n_{u} \times n_{y}}$ and $P \in \mathcal{F}^{n_{y} \times n_{u}}$ represent the controller and the plant respectivelly. Denote by $H(P, K)$ the transfer function matrix from $\left[\begin{array}{ll}\nu_{2}^{T} & \nu_{1}^{T}\end{array}\right]^{T}$ to $\left[\begin{array}{ll}y^{T} & u^{T}\end{array}\right]^{T}$ (provided that $(I+K P)$ is nonsingular):

$$
H(P, K)=\left[\begin{array}{cc}
(I+P K)^{-1} & -P(I+K P)^{-1} \\
K(I+P K)^{-1} & (I+K P)^{-1}
\end{array}\right]
$$

Analogously with the generalized-feedback system, if the transfer matrix $H(P, K)$ is over $\mathcal{A}$ we call it stable, or we say that $K$ is a stabilizing controller of $P$ or equivalently that $K$ stabilizes $P$. If a stabilizing controller of $P$ exists, we say that $P$ is stabilizable. The following Lemma from [4] is a generalization of the well-known Theorem 4.3.2 in [1].

Lemma 3.1: [4, Lemma 1] Let $G$ and $K$ be a generalized plant and its controller over $\mathcal{F}$, with $G$ stabilizable. Then $\Theta(G, K)$ is stable if and only if $H\left(-G_{y u}, K\right)$ is stable.
We define the set $\mathcal{C}$ of stabilizing controllers of $P$ by

$$
\mathcal{C} \stackrel{\text { def }}{=}\left\{K \mid K \in \mathcal{F}^{n_{y} \times n_{u}} \text { and } K \text { stablilizes } P\right\} .
$$

Clearly, from Lemma 3.1, the set $\mathcal{C}$ can be recast as

$$
\mathcal{C}=\left\{K \mid K \in \mathcal{F}^{n_{y} \times n_{u}} \text { and } K \text { stabilizes } G\right\},
$$

for any generalized plant $G$ for which $G_{y u}=-P$.

\section{A. The coordinate free parametrization}

Of central importance in the sequel is the result stated below (a summary of Theorem 4.2 and Theorem 4.3 in [3]) as it provides a useful parametrization of the stabilizing controllers of $H(P, K)$.

Theorem 3.2: [3] i) Given the integers $n_{u}$ and $n_{y}$, and a plant $P$ in the set $\mathcal{F}^{n_{y} \times n_{u}}$, consider the following set:

$\mathcal{H}_{P} \stackrel{\text { def }}{=}\left\{H(P, K) \in \mathcal{A}^{\left(n_{u}+n_{y}\right) \times\left(n_{u}+n_{y}\right)} \mid\right.$

$K$ is a stabilizing controller for $P\}$.

Given an arbitrary $K_{0}$ that stabilizes $P$, and therefore for which $H\left(P, K_{0}\right) \in \mathcal{A}^{\left(n_{u}+n_{y}\right) \times\left(n_{u}+n_{y}\right)}$, the following holds: $\mathcal{H}_{P}=\left\{\Omega(Q) \mid Q \in \mathcal{A}^{\left(n_{u}+n_{y}\right) \times\left(n_{u}+n_{y}\right)}\right.$,

with $Q$ causal and $\Omega(Q)$ nonsingular $\}$ where for any causal $Q$ in the set $\mathcal{A}^{\left(n_{u}+n_{y}\right) \times\left(n_{u}+n_{y}\right)}, \Omega(Q)$ is defined as follows:

$$
\begin{aligned}
\Omega(Q) & \stackrel{\text { def }}{=}\left(H\left(P, K_{0}\right)-\left[\begin{array}{cc}
I_{n_{y}} & O \\
O & O
\end{array}\right]\right) Q \times \\
& \times\left(H\left(P, K_{0}\right)-\left[\begin{array}{cc}
O & O \\
O & I_{n_{u}}
\end{array}\right]\right)+H\left(P, K_{0}\right) .
\end{aligned}
$$

Here $I_{n_{y}}$ and $I_{n_{u}}$ denote the identity matrices of dimension $n_{y}$ and $n_{u}$ respectively.

ii) For $\Omega(Q)$ defined in (4), belonging to the set $\mathcal{A}^{\left(n_{u}+n_{y}\right) \times\left(n_{u}+n_{y}\right)}$, consider the conformal partition defined as follows:

$$
\left.\Omega(Q)=\left[\begin{array}{ll}
\Omega_{\Omega_{11}(Q)} & \overbrace{\Omega_{12}(Q)}^{n_{y}} \\
\Omega_{21}(Q) & n_{22}(Q)
\end{array}\right]\right\} \begin{aligned}
& n_{y} \\
& n_{u}
\end{aligned}
$$

If $\Omega_{11}(Q)$ is nonsingular then any stabilizing controller of $P$ can be written as

$$
K(Q)=\Omega_{21}(Q) \Omega_{11}^{-1}(Q)
$$

for some causal matrix $Q$ in the set $\mathcal{A}^{\left(n_{u}+n_{y}\right) \times\left(n_{u}+n_{y}\right)}$, where $\Omega_{21}$ and $\Omega_{11}$ are the $(2,1)$ - and $(1,1)$ - blocks of $\Omega(Q)$ respectively.

Remark 3.1: We must point out here that the frequently invoked reference [4] contains a typo which is repeated for several times throughout the paper. Specifically, in Section III of [4], the expression of $K$ is given as $\Omega_{21}(Q) \Omega_{22}^{-1}(Q)$ for some causal $Q$ in $\mathcal{A}^{\left(n_{u}+n_{y}\right) \times\left(n_{u}+n_{y}\right)}$. Accordingly to [3, Proposition 5] (and as implied by (2) and Theorem 3.2 ), the correct expression for the stabilizing controller is given by $\Omega_{21}(Q) \Omega_{11}^{-1}(Q)$ or $\Omega_{22}^{-1}(Q) \Omega_{21}(Q)$ for some causal, stable $Q$. References to the results in [4] are made in the sequel, taking into account the aforementioned typo. 


\section{B. The strict causality assumption.}

From this point onward we shall make the hypothesis on $G_{y u}$ to be strictly causal. This is necessary as to guarantee several conditions in a way made precise by the following remarks.

Remark 3.2: [4] The assumption of strict causality of $G_{y u}$ implies that every stabilizing controller is causal [2, Proposition 6.2] and that the closed loop is well-posed [9, pp.119] for every stabilizing controller [3, Proposition 5].

Remark 3.3: [3, Proposition 5] The assumption of strict causality on $G_{y u}$ also makes superfluous the conditions from Theorem 3.2 on the invertibility of $\Omega(Q)$ and $\Omega_{11}(Q)$, meaning that under the strict causality hypothesis of $G_{y u}$, the $\Omega(Q), \Omega_{11}(Q)$ (and $\Omega_{22}(Q)$ ) are invertible for any $Q$ causal and stable.

Remark 3.4: Using Lemma 3.1, we conclude that Theorem 3.2 ii) provides a parametrization of all stabilizing controllers of $\Theta(G, K)$, for any generalized plant $G$ for which $G_{y u}=-P$.

\section{Problem Formulation}

\section{A. The Standard Control Problem}

Assume that a consistent norm has been adopted for transfer matrices over $\mathcal{F}$. A standard problem in control is the following: in the generalized feedback system from Figure 1, with the given causal and stabilizable plant matrix $G$, design a stabilizing controller $K$ that minimizes the norm of the top left corner entry of $\Theta(G, K)$ which is the transfer function from $w$ to $z$, namely

$$
\min \quad\left\|G_{z w}+G_{z u} K\left(I-G_{y u} K\right)^{-1} G_{y w}\right\| .
$$

$K$ stabilizes $G$

The following result, [4, Theorem 1] will be instrumental in our proposed approach, as it makes clear the equivalence between the standard control problem (7) and the modelmatching problem of minimizing the norm of some affine (and therefore convex) function in the argument $Q$ - the parameter from Theorem 3.2.

Theorem 4.1: [4, Theorem 1] Let $G$ be a stabilizable, generalized plant such that the block $G_{y u} \in \mathcal{F}^{n_{y} \times n_{u}}$ is strictly causal. Given any stabilizable controller $K_{0} \in \mathcal{F}^{n_{u} \times n_{y}}$ of $G_{y u}$, the standard control problem (7) is equivalent to

$$
\min _{Q \in \mathcal{A}^{\left(n_{u}+n_{y}\right) \times\left(n_{u}+n_{y}\right)}}\left\|T_{1}-T_{2} Q T_{3}\right\|
$$

subject to $Q$ causal and stable, where an optimal solution $K^{*}$ to (7) can always be obtained from the optimal $Q$ in (8), denoted with $Q^{*}$, via $K^{*}=\Omega_{21}\left(Q^{*}\right) \Omega_{11}^{-1}\left(Q^{*}\right)$. Here $T_{1}, T_{2}$ and $T_{3}$ are the transfer function matrices defined below:

$$
\begin{aligned}
& T_{1} \stackrel{\text { def }}{=} G_{z w}+G_{z u} K_{0}\left(I-G_{y u} K_{0}\right)^{-1} G_{y w}, \\
& T_{2} \stackrel{\text { def }}{=}\left[\begin{array}{cc}
G_{z u} K_{0}\left(I-G_{y u} K_{0}\right)^{-1} & G_{z u}\left(I-K_{0} G_{y u}\right)^{-1}
\end{array}\right], \\
& T_{3} \stackrel{\text { def }}{=}\left[\begin{array}{c}
\left(I-G_{y u} K_{0}\right)^{-1} G_{y w} \\
K_{0}\left(I-G_{y u} K_{0}\right)^{-1} G_{y w}
\end{array}\right] .
\end{aligned}
$$

\section{B. The Decentralized Control Problem}

Notation: For $p \geq 1$, we denote the set of integers from 1 to $p$ with $\overline{1, p}$. Throughout the sequel we consider that the transfer function matrix $G_{y u} \in \mathcal{F}^{n_{y} \times n_{u}}$ is partitioned in $p$ block-rows and $m$ block-columns. The $i$-th block-row has $n_{y}^{i}$ rows, while the $j$-th block-column has $n_{u}^{j}$ columns. Obviously, $\sum_{i=1}^{p} n_{y}^{i}=n_{y}$ and $\sum_{j=1}^{m} n_{u}^{j}=n_{u}$. For every pair $(i, j)$ in the set $\overline{1, p} \times \overline{1, m}$, we denote by $\left[G_{y u}\right]_{i j} \in$ $\mathcal{F}^{n_{y}^{i} \times n_{u}^{j}}$ the transfer matrix at the intersection of the $i$-th block-row and $j$-th block-column of $G_{y u}$. Accordingly,

$$
G_{y u}=\left[\begin{array}{ccc}
{\left[G_{y u}\right]_{11}} & \cdots & {\left[G_{y u}\right]_{1 m}} \\
\vdots & & \vdots \\
{\left[G_{y u}\right]_{p 1}} & \cdots & {\left[G_{y u}\right]_{p m}}
\end{array}\right]
$$

with $\left[G_{y u}\right]_{i j} \in \mathcal{F}^{n_{y}^{i} \times n_{u}^{j}}$. Henceforth, we shall use this square bracketed notation for block indexing of transfer function matrices.

Analogously, the controller's transfer function matrix $K \in$ $\mathcal{F}^{n_{u} \times n_{y}}$ is partitioned in $m$ block-rows and $p$ blockcolumns, where the $j$-th block-row has $n_{u}^{j}$ rows and the $i$-th block-column has $n_{y}^{i}$ columns. Correspondingly, $[K]_{j i}$ is the notation for the element of $\mathcal{F}^{n_{u}^{j} \times n_{y}^{i}}$ at the intersection of the $j$-th block-row and $i$-th block-column of $K$.

The decentralized setting will be modeled throughout the paper via the sparsity constraints paradigm, as it has been proved to be a suitable method to formalize many problems in decentralized control. The notation we introduce next is entirely concordant with the one used in [5] and [6] to define the sparsity constraints.

For the $\{0,1\}$ boolean algebra the operations $(+, \cdot)$ are defined as usual: $0+0=0 \cdot 1=1 \cdot 0=0 \cdot 0=0$ and $1+0=0+1=1+1=1 \cdot 1=1$. By a binary matrix we mean a matrix whose entries belong to the set $\{0,1\}$. Naturally, the addition and multiplication of binary matrices is carried out over the Boolean algebra and under the aforementioned assumptions, the addition and multiplication of binary matrices matrices are defined as in the real case.

Binary matrices will be denoted by capital letters with the "bin" superscript, in order to be distinguished from transfer function matrices over $\mathcal{F}$ which are represented in the sequel by plain capital letters. Furthermore, for binary matrices only, the notation $A^{\text {bin }} \leq B^{\text {bin }}$ means that $a_{i j} \leq b_{i j}$ for all $i$ and $j$, that is for all the entries of $A^{\text {bin }}$ and $B^{\text {bin }}$ respectively.

Henceforth, we adopt the convention that the transfer function matrices are indexed by blocks while binary matrices are indexed by each individual entry. 
For any $K \in \mathcal{F}^{n_{u} \times n_{y}}$ define $\operatorname{Pattern}(K) \in\{0,1\}^{m \times p}$ to be the binary matrix given by

$$
\operatorname{Pattern}(K)_{i j} \stackrel{\text { def }}{=} \begin{cases}0 & \text { if the block }[K]_{i j}=0 ; \\ 1 & \text { otherwise }\end{cases}
$$

Accordingly, the binary value of Pattern $(K)_{k l}$ determines whether controller $K$ may use measurements from the output of the system $l$, since $[K]_{k l}$ is the map from the outputs of subsystem $l$ to the inputs of subsystem $k$, while $\left[G_{y u}\right]_{i j}$ represents the map from the inputs of subsystem $j$ to the outputs of subsystem $i$.

Conversely, for any binary matrix with $m$ rows and $p$ columns $K^{\text {bin }} \in\{0,1\}^{m \times p}$, we can define the following linear subspace of $\mathcal{F}^{n_{u} \times n_{y}}$ :

$$
\operatorname{Sparse}\left(K^{\mathrm{bin}}\right) \stackrel{\text { def }}{=}\left\{K \in \mathcal{F}^{n_{u} \times n_{y}} \mid \operatorname{Pattern}(K)=K^{\mathrm{bin}}\right\}
$$

Hence $\operatorname{Sparse}\left(K^{\text {bin }}\right)$ is the set of all controllers $K$ in $\mathcal{F}^{n_{u} \times n_{y}}$ for which $[K]_{i j}=0$ whenever $K_{i j}^{\text {bin }}=0$, where by $[K]_{i j}=0$ we mean that the $(i, j)$-th block of $K$ is the zero matrix.

Let $K^{\text {bin }} \in\{0,1\}^{m \times p}$ be the requested sparsity pattern of the controller. Define the subset $\mathcal{S}$ of $\mathcal{F}^{n_{u} \times n_{y}}$ as

$$
\mathcal{S} \stackrel{\text { def }}{=} \operatorname{Sparse}\left(K^{\mathrm{bin}}\right)
$$

as the set of transfer function matrices that satisfy the cotroller's imposed sparsity structure. Similarly, $P^{\text {bin }}$ in the

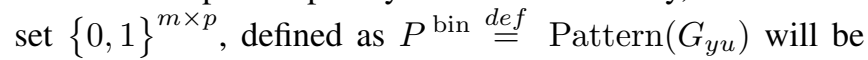
the sparsity pattern of the $G_{y u}$ block of the generalized plant.

The Decentralized Problem via Sparsity Constraints. We are ready now for the main result of this subsection. Suppose that the generalized plant $G$ is stabilizable with a controller $K_{0} \in \mathcal{S}$. The decentralized version of the standard problem (7) is formulated by simply adding the extra constraint $K \in$ $\mathcal{S}$, specifically

$$
\min _{\substack{K \text { stabilizes } G \\ K \in \mathcal{S}}}\left\|G_{z w}+G_{z u} K\left(I-G_{y u} K\right)^{-1} G_{y w}\right\| .
$$

The following result (in fact a Corollary of Theorem 4.1) is central in our proposed approach:

Corollary 4.2: Let $G$ be a stabilizable, generalized plant such that the block $G_{y u} \in \mathcal{F}^{n_{y} \times n_{u}}$ is strictly causal. Given any stabilizing controller $K_{0} \in \mathcal{S}$ of $G_{y u}$, the decentralized control problem (13) is equivalent to

$$
\min _{\substack{Q \in \mathcal{A}^{\left(n_{u}+n_{y}\right) \times\left(n_{u}+n_{y}\right)} \\ \Omega_{21}(Q) \Omega_{11}^{-1}(Q) \in \mathcal{S}}}^{\left\|T_{1}-T_{2} Q T_{3}\right\| .}
$$

where an optimal solution $K^{*}$ to (13) can always be obtained from the optimal $Q$ in (14), denoted with $Q^{*}$, via $K^{*}=$ $\Omega_{21}\left(Q^{*}\right) \Omega_{11}^{-1}\left(Q^{*}\right)$. Here, $T_{1}, T_{2}$ and $T_{3}$ are as in (9).
Proof: As mentioned in subsection III-B, throughout the paper we are under the assumption that the $G_{y u}$ block is strictly causal. This assumption has the desirable feature of ensuring that (see Remark 3.2) every stabilizing controller is causal and that the closed loop is well-posed for any stabilizing controller. Moreover, it also ensures (see Remark 3.3) that $\Omega(Q)$ and $\Omega_{11}(Q)$ are nonsigular for any causal $Q$ in the set $\mathcal{A}^{\left(n_{u}+n_{y}\right) \times\left(n_{u}+n_{y}\right)}$.

This Corollary makes clear that problems (13) and (14) are equivalent since they are obtained by simply adding the additional constraint $K \in \mathcal{S}$ to the equivalent problems (via Theorem 4.1) (7) and (8) respectively. The constraint $K \in \mathcal{S}$ arises from the imposed decentralized structure of the controller.

Suppose that $K$ is a solution of (13). Theorem 3.2 ii) implies that for any stabilizing controller $K$ in the set $\mathcal{S}$, there exists a $Q \in \mathcal{A}^{\left(n_{u}+n_{y}\right) \times\left(n_{u}+n_{y}\right)}$ such that $K=$ $\Omega_{21}(Q) \Omega_{11}^{-1}(Q)$ and therefore $\Omega_{21}(Q) \Omega_{11}^{-1}(Q) \in \mathcal{S}$. The rest follows from Theorem 4.1) on the equivalence between (7) and (8).

Conversely, in (14) for any $Q \in \mathcal{A}^{\left(n_{u}+n_{y}\right) \times\left(n_{u}+n_{y}\right)}$ it follows also by Theorem 3.2 that $\Omega_{21}(Q) \Omega_{11}^{-1}(Q)$ is a stabilizing controller of $G_{y u}$. Hence the constraint $\Omega_{21}(Q) \Omega_{11}^{-1}(Q) \in \mathcal{S}$ being equivalent to the constraint $K \in \mathcal{S}$ in (13), yields the decentralized model-matching problem (14).

\section{Sparsity Constraints on the Q-Parameter}

The binary matrix $K^{\text {bin }}$ in the set $\{0,1\}^{m \times p}$ will denote from now on the sparsity pattern of the adissible decentralized controllers. Similarly, $P^{\text {bin }}$ in the set $\{0,1\}^{p \times m}$ is the sparsity structure of the $G_{y u}$ block of $G$. Consider now the following natural partition of the parameter $Q$, element of $\mathcal{A}^{\left(n_{u}+n_{y}\right) \times\left(n_{u}+n_{y}\right)}$ :

$$
\left.Q=\left[\begin{array}{ll}
\overbrace{Q_{11}}^{n_{y}} & \overbrace{Q_{12}}^{n_{u}} \\
Q_{21} & Q_{22}
\end{array}\right]\right\} n_{y}
$$

Assume for $Q_{12} \in \mathcal{A}^{n_{y} \times n_{u}}$ the same partition by blocks as for $G_{y u}$, from the beginning of the previous subsection. That is, $Q_{12}$ is partitioned in $p$ block-rows and $m$ blockcolumns and the $i$-th block-row has $n_{y}^{i}$ rows, while the $j$ th block-column has $n_{u}^{j}$ columns. Hence for any $(i, j) \in$ $\overline{1, p} \times \overline{1, m}$ we get that $\left[Q_{12}\right]_{i j} \in \mathcal{A}^{n_{y}^{i} \times n_{u}^{j}}$. Similarly, assume for $Q_{21} \in \mathcal{A}^{n_{u} \times n_{y}}$ the same partition by blocks as for $K: m$ block-rows and $p$ block-columns and for any $(j, i) \in \overline{1, m} \times \overline{1, p},\left[Q_{21}\right]_{j i} \in \mathcal{A}^{n_{u}^{j} \times n_{y}^{i}}$. It follows that $Q_{11}$ is naturally partitioned in $p$ block-rows times $p$ blockcolumns and the $i$-th block-row has $n_{y}^{i}$ rows, while the $i$-th block-column has $n_{y}^{i}$ columns. Consequently, for any $(i, j) \in \overline{1, p} \times \overline{1, p}$ we get that $\left[Q_{11}\right]_{i j} \in \mathcal{A}^{n_{y}^{i} \times n_{y}^{j}}$. Similarly, $Q_{22}$ has $m$ block-rows and $m$ block-columns and the $j$-th block-row has $n_{u}^{j}$ rows, while the $j$-th block-column has $n_{u}^{j}$ columns. 
For the transfer function matrices $Q_{11}, Q_{12}, Q_{21}$, and $Q_{22}$ we define next their corresponding sparsity patterns. As defined in (10), the function Pattern (.) is referred in (16) below via block indexation.

$$
\begin{aligned}
& \mathbb{Q}_{11} \stackrel{\text { def }}{=}\left\{Q_{11} \in \mathcal{A}^{n_{y} \times n_{y}} \mid \text { Pattern }\left(Q_{11}\right)=\right. \\
& \left.=P^{\text {bin }} K^{\text {bin }}+I_{m}\right\} \text {, } \\
& \mathbb{Q}_{12} \stackrel{\text { def }}{=}\left\{Q_{12} \in \mathcal{A}^{n_{y} \times n_{u}} \mid \text { Pattern }\left(Q_{12}\right)=\right. \\
& \left.=P^{\text {bin }} K^{\text {bin }} P^{\text {bin }}+P^{\text {bin }}\right\} \text {, } \\
& \mathbb{Q}_{21} \stackrel{\text { def }}{=}\left\{Q_{21} \in \mathcal{A}^{n_{u} \times n_{y}} \mid \text { Pattern }\left(Q_{21}\right)=K^{\text {bin }}\right\}, \\
& \mathbb{Q}_{22} \stackrel{\text { def }}{=}\left\{Q_{22} \in \mathcal{A}^{n_{u} \times n_{u}} \mid \text { Pattern }\left(Q_{22}\right)=K^{\text {bin }} P^{\text {bin }}\right\} \text {. }
\end{aligned}
$$

Next, define the subset of $\mathcal{A}^{\left(n_{u}+n_{y}\right) \times\left(n_{u}+n_{y}\right)}$

$$
\begin{gathered}
\mathbb{Q} \stackrel{\text { def }}{=}\left\{\left[\begin{array}{ll}
Q_{11} & Q_{12} \\
Q_{21} & Q_{22}
\end{array}\right] \in \mathcal{A}^{\left(n_{u}+n_{y}\right) \times\left(n_{u}+n_{y}\right)} \mid\right. \\
\left.\mid Q_{11} \in \mathbb{Q}_{11}, Q_{12} \in \mathbb{Q}_{12}, Q_{21} \in \mathbb{Q}_{21}, Q_{22} \in \mathbb{Q}_{22}\right\} .
\end{gathered}
$$

Define the following binary matrix in the set $\{0,1\}^{(p+m) \times(p+m)}$

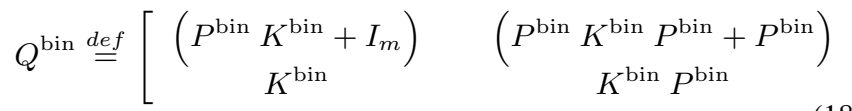

With this we get the following equivalent characterization of the set $\mathbb{Q}$ from (17):

$$
\mathbb{Q}=\left\{Q \in \mathcal{A}^{\left(n_{u}+n_{y}\right) \times\left(n_{u}+n_{y}\right)} \mid \operatorname{Pattern}(Q)=Q^{\text {bin }}\right\} .
$$

Remark 4.1: The alternative characterization of $\mathbb{Q}$ from (19) points out that $\mathbb{Q}$ is perfectly defined solely by the sparsity matrix $Q^{\text {bin }}$. The set $\mathbb{Q}$, contains only those square, stable parameters $Q$ from the set $\mathcal{A}^{\left(n_{u}+n_{y}\right) \times\left(n_{u}+n_{y}\right)}$, that have the specific sparsity pattern induced by $Q^{\text {bin }}$.

\section{Quadratic Invariance}

From this point on, we take into account the Banach space structure of the linear spaces $\mathcal{F}^{n_{u}}$ and $\mathcal{F}^{n_{y}}$. For this, we can consider for instance the $\mathcal{H}^{2}$ or $\mathcal{H}^{\infty}$ norms. The definition of these norms for matrices with entries real rational functions of multivariate polynomials is done by a natural extension from the classical case of real rational matrix functions (which are the input/output operator of LTI systems).

Furthermore, under these assumptions, the set $\mathcal{S}$ defined in (12), is a closed linear subspace of $\mathcal{F}^{n_{u} \times n_{y}}$.

We restate next the definition of quadratic invariance of the set $\mathcal{S}$ with respect to the plant $G_{y u}$, firstly introduced in [6] (Definition 2, pp. 277), [5], [7], as this is among the hypotheses of our main result.
Definition 4.1: The set $\mathcal{S}$ is called quadratically invariant with respect to the plant $G_{y u}$ if

$$
K G_{y u} K \in \mathcal{S} \quad \text { for all } K \in \mathcal{S} .
$$

\section{MAin RESUlT}

As stated in Theorem 3.2 (see also Lemma 3.1), any stabilizing controller $K$ of $G$ has the form

$$
K(Q) \stackrel{(6)}{=} \Omega_{21}(Q) \Omega_{11}^{-1}(Q)=\Omega_{21}(Q)\left(I-P \Omega_{21}(Q)\right)^{-1}
$$

for some stable $Q \in \mathcal{A}^{\left(n_{u}+n_{y}\right) \times\left(n_{u}+n_{y}\right)}$. In a somehow similar manner with how the strongly-stabilizable case was dealt with in [6], in this section we prove that the information constraints on the controller $(K \in \mathcal{S})$, are equivalent to constraints on the parameter $Q$ (namely that $Q \in \mathbb{Q}$ ). More specifically, if we impose the constraint $K \in \mathcal{S}$ then any such stabilizing controller $K(Q)$ of $G$ is going to be of the form $K(Q)=\Omega_{21}(Q)\left(I-P \Omega_{21}(Q)\right)^{-1}$ and it will belong to the set $(\mathcal{C} \cap \mathcal{S})$ for some $Q \in \mathbb{Q}$. These important facts are precisely stated in Lemma 5.2 and Theorem 5.3.

We summarize here the hypothesis and notations that we assume for our main result:

- The set $\mathcal{F}^{n \times r}$ of transfer functions matrices over $\mathcal{F}$ along with the invoked norm is a Banach space. (This will hold true for all the particular instances of $\mathcal{A}$ that we are interested in);

- The given generalized plant $G$ is stabilizable by a controller $K_{0} \in \mathcal{S}$;

- The block $G_{y u}$ is strictly causal (see Remark 3.2);

- The set $\mathcal{S}$ is quadratically invariant under $G_{y u}$;

- We will denote by $P$ the block $\left(-G_{y u}\right)$ as we refer to it repeatedly, hence $P \stackrel{\text { def }}{=}-G_{y u}$.

The following preliminary result will be needed in the proof of the main result.

Proposition 5.1: For any $Q \in \mathcal{A}^{\left(n_{u}+n_{y}\right) \times\left(n_{u}+n_{y}\right)}$, the first block column of $\Omega(Q)$ from (5) has the following expression:

$$
\begin{gathered}
{\left[\begin{array}{l}
\Omega_{11}(Q) \\
\Omega_{21}(Q)
\end{array}\right]=\left[\begin{array}{c}
I_{n_{y}}-P \Omega_{21}(Q) \\
\Omega_{21}(Q)
\end{array}\right] \text { where }} \\
\Omega_{21}(Q)=\left(I+K_{0} P\right)^{-1}\left(K_{0}+K_{0} P K_{0}+K_{0} Q_{11}+\right. \\
\left.+K_{0} Q_{12} K_{0}+Q_{21}+Q_{22} K_{0}\right)\left(I+P K_{0}\right)^{-1}
\end{gathered}
$$

Proof. The proof is purely algebraic, for details see [10].

Lemma 5.2: Let $G$ be a causal, generalized plant stabilizable with a controller $K_{0} \in \mathcal{S}$. Assume that $\mathcal{S}$ is quadratically invariant under the strictly causal block $P$. The function $K: \mathbb{Q} \longmapsto(\mathcal{C} \cap \mathcal{S})$ with $K(Q) \stackrel{\text { def }}{=} \Omega_{21}(Q) \Omega_{11}^{-1}(Q)$ is onto.

Proof. The proof is made two steps. First it is shown that $K(\cdot)$ is a well-defined function from $\mathbb{Q}$ to $\mathcal{C} \cap \mathcal{S}$, then it is proved that this function is onto. The proof is 
entirely algebraic and essentially consists in employing the quadratically invariance hypothesis to show that each of the terms in the sum on the right hand side of (23) belongs to $\mathcal{S}$. For details we refer to [10].

The previous Lemma is the centerpiece of our main result, as it proves that the constraint $K(Q) \in \mathcal{S}$ (equivalent via (6) with $\Omega_{21}(Q) \Omega_{11}^{-1}(Q) \in \mathcal{S}$ ) is actually equivalent in problem (14) with the constraint $Q \in \mathbb{Q}$.

Theorem 5.3: Let $G$ be a causal, generalized plant stabilizable with a controller $K_{0} \in \mathcal{S}$. Assume that $\mathcal{S}$ is quadratically invariant under the strictly causal block $P$. The decentralized optimal control problem (13) is equivalent with the problem

$$
\min _{Q \in \mathbb{Q}}\left\|T_{1}-T_{2} Q T_{3}\right\| .
$$

where $T_{1}, T_{2}$ and $T_{3}$ are as in (9). An optimal solution $K^{*}$ to (13) can always be obtained from the optimal $Q$ in (24), denoted with $Q^{*}$, via $K^{*}=\Omega_{21}\left(Q^{*}\right) \Omega_{11}^{-1}\left(Q^{*}\right)$. Here, $\Omega_{21}(Q)$ and $\Omega_{11}(Q)$ are as in (23),(22).

Proof: It follows from Corollary 4.2 and the previous Lemma.

The convex problem (24) is similar with problem (6) pp. 281 in [6] which is the equivalent convex problem to solve the decentralized optimal $\mathcal{H}^{2}$ synthesis for strongly stabilizable plants. If we consider $\mathcal{A}=\mathcal{R} \mathcal{H}^{2}$ then the vectorization techniques from Section VI of [6] are readily applicable for solving (24) and get the optimal $\mathcal{H}^{2}$ controller, without any strong-stabilizability assumption.

\section{ACKNOWLEDGMENTS}

Both authors are very much in debt to Michael Rotkowitz for useful discussions and comments on the manuscript.

\section{REFERENCES}

[1] B. Francis, “ A Course in $\mathcal{H}_{\infty}$ Control Theory", Series Lecture Notes in Control and Information Sciences, New York: Springer-Verlag, 1987, vol. 88.

[2] K. Mori and K. Abe, " Feedback Stabilization over Commutative Rings: Further Study of Coordinate-free Approach", SIAM J. Control Optim., Vol. 39, No. 6, pp 1952-1973, 2001.

[3] K. Mori "Paramaetrization of Stabilizing Controllers over Commutative Rings with Applications to Multidimensional Systems", IEEE Trans. Circuits Syst. I, Vol. 49, 2002. (pp. 743-752)

[4] K. Mori "Relationship Between Standard Control Problem and ModelMatching Problem Without Coprime Factorizability", IEEE Trans. Aut. Control, Vol. 49, No.2, 2004. (pp. 230-233)

[5] M. Rotkowitz, "Tractable Problems in Optimal Decentralized Control", Ph.D. disertation, Stanford Univ., Stanford CA, Jun 2005.

[6] M. Rotkowitz, S. Lall "A Characterization of Convex Problems in Decentralized Control", IEEE Trans. Aut. Control, Vol.51, No.2, 2006. (pp. 274-286)

[7] M. Rotkowitz, S. Lall "Affine Controller Parametrization for Decentralized Control Over Banach Spaces", IEEE Trans. Aut. Control, Vol.51, No.9, 2006. (pp. 1497-1500)

[8] M. Vidyasagar, H. Schneider, B. Francis "Algebraic and Topological Aspects of Feedback Stabilization", IEEE Trans. Aut. Control, Vol. 27, No. 4, 1982. (pp. 880-894)

[9] K. Zhou, J.C. Doyle and K. Glover, " Robust and Optimal Control”, Upper Saddle River, NJ: Prentice Hall, 1996.
[10] Ş. Sabău, N.C. Martins, M.C. Rotkowitz, "A convex parameterization of all stabilizing controllers for non-strongly stabilizable plants, under quadratically invariant sparsity constraints", I.S.R. Technichal Report. Available at:

http://www.lib.umd.edu/drum/bitstream/

1903/8688/3/NonStrStabEdited.pdf 